\title{
Low energy structures of gold nanoclusters in the size range 3 to
}

\section{8 atoms}

\author{
B. Soulé de Bas, M. J. Ford, and M. B. Cortie \\ Institute for Nanoscale Technology, University of Technology, Sydney, PO Box 123, \\ Broadway, NSW 2007, Australia
}

\begin{abstract}
Using a combination of first principles calculations and empirical potentials we have undertaken a systematic study of the low energy structures of gold nanoclusters containing from 3 to 38 atoms. A Lennard-Jones and many-body potential have been used in the empirical calculations, while the first principles calculations employ an atomic orbital, density functional technique. For the smaller clusters $(n=3,4$ and 5$)$ the potential energy surface has been mapped at the $a b$ initio level, for larger clusters an empirical potential was first used to identify low energy candidates which were then optimised with full $a b$ initio calculations. At the DFT-LDA level, planar structures persist up to 6 atoms and are considerably more stable than the cage structures by more than $0.1 \mathrm{eV} /$ atom. The difference in $a b$ initio energy between the most stable planar and cage structures for 7 atoms is only $0.04 \mathrm{eV} /$ atom. For larger clusters there are generally a number of minima in the potential energy surface lying very close in energy. Furthermore our calculations do not predict ordered structures for the magic numbers $\mathrm{n}=$ 13 and 38. They do predict the ordered tetrahedral structure for $n=20$. The results of the calculations show that gold nanoclusters in this size range are mainly disordered and will likely exist in a range of structures at room temperature.
\end{abstract}


Keywords: Au, DFT, PES, Isomers

\section{INTRODUCTION}

There has been considerable interest in gold nanoclusters in recent years motivated in part by the catalytic activity of small supported clusters [1]. Their increasing application as nanoscale building blocks in materials and devices [2] and their unique optical properties [3] have also motivated much of this work. A fundamental understanding of the physical and chemical properties of clusters, and in particular the size dependence of these properties, is central to developing many of these applications. Systematic structural studies of clusters over varying size ranges play an important role as structure represents the starting point for understanding other cluster properties and may indeed be a determining factor in their physical and chemical behaviour.

Generally, the applications alluded to above involve clusters supported on a solid surface, or clusters containing 1000's of atoms, which are additionally surface passivated. Although the ultimate aim of computational efforts may be to study such complex systems, initial efforts must proceed from far simpler systems. Theoretical determination of the equilibrium structures from first principles rapidly becomes intractable with increasing cluster size, first because of the computational resources required, and second because determination of global minima rapidly becomes difficult as the number of degrees of freedom of the potential energy surface increases. The second factor is particularly apparent for gold clusters where the number of local minima grows rapidly due to the short range nature of the gold-gold interaction. Consequently there still exists debate over theoretical minimum energy structures even for the smallest clusters 
containing 10's of atoms. This problem is further compounded by the difficulty of direct experimental structure determination using diffraction, electron microscopy and scanned probe techniques in this size range. Cluster structures can, to some extent, be inferred from measuring structure dependent properties such as detachment energies by photoelectron spectroscopy. This approach is, however, yet to yield definitive low energy structures. In the present work we have attempted to systematically map the potential energy surfaces using a combination of empirical potential and first principles calculations for gold clusters containing between 3 and 38 atoms. In addition, we also investigated specific ordered structures likely to have low energies. The aim is to provide a comprehensive study of structures in order to shed light on the current controversies. In the longer term we are interested in modelling gold nanoparticles representative of experimental measurements and applications, that is large clusters which are surface passivated or supported on a substrate.

A number of important factors have emerged from recent theoretical calculations of gold nanoparticles. As mentioned above the potential energy surfaces are complex and contain many local minima. An extensive search of the PES with no symmetry constraints is required in order to locate global minima. Some of the earlier studies, although conducted using high level $a b$ initio techniques were restricted to fixed symmetries and so excluded the possibility of dis-ordered structures [4]. The use of empirical potentials such as embedded atom or Sutton-Chen potentials make searching the PES more feasible, however the energy ordering of the large number of local minima, and whether ordered or disordered structures are most stable can be quite sensitive to the form of the empirical potential that is used. Recent studies using the $n$-body Gupta 
potential and first principles DFT calculations for gold clusters containing 38, 55 or 78 atoms provide evidence that for these magic numbers the most stable structure is disordered but lies close in energy to crystalline or quasi-crystalline structures [5-7]. Even for the much smaller magic number clusters containing 13 and 19 atoms, Wang et al [8] find a preferred disordered structure rather than the compact ordered icosahedral or fcclike structure. However, ordered structures cannot be excluded as shown by Li et al [9] who found the tetrahedron as the lowest energy structure for $\mathrm{Au}_{20}$ based upon a theoretical and experimental investigation.

Strong relativistic effects occur in the interactions between gold atoms which influence the stability of various structures. Hakkinen and Landman [10] have performed DFT calculations using a scalar relativistic pseudopotential for clusters between 2 and 10 atoms and find that flat 2D structures persist up to 7 atoms. Bonačić-Koutecký et al. [11] and Lee et al. [12], using DFT with relativistic pseudopotentials, predict this transition from planar to 3D structure at 10 and 13 atoms respectively. On the other hand Wang et al [8] report this transition at a smaller size, between $\mathrm{Au}_{6}$ and $\mathrm{Au}_{7}$, The latter calculations also employ a core pseudopotential corrected for relativistic effects. Finally, it is interesting to note that more recent calculations by Hakkinen et al [13], for small Au anion and neutral clusters, predict planar structures stable up to 12 atoms for $\mathrm{Au}_{\mathrm{n}}{ }^{-}$and 13 atoms for $\mathrm{Au}_{\mathrm{n}}$. It is clear from these results that the planar-3D transition maybe sensitive to other factors beyond relativistic ones.

\section{METHODS}


In the present work we have used a combination of empirical potentials and $a b$ initio calculations to locate global minimum energy structures and low-lying isomers. For clusters containing fewer than 6 atoms it is possible to exhaustively search the potential energy surface (PES) to identify the equilibrium structures, stable isomers, and activation barriers between structures. For $n=3$ to 5 , the PES was systematically mapped in a series of single-point DFT calculations. The resulting minimum energy structures were then further refined by a conjugate-gradient relaxation. However, above $n=5$, the number of degrees of freedom rapidly increases making potential energy scans problematic, particularly for clusters of gold atoms where the number of low-lying minima can be large. Hence, for larger clusters we use an empirical potential to locate minima in the PES rapidly and then relax the resulting structures using density functional calculations. However this method has limitations because of the strong dependence of the PES search on the empirical potential used. In addition to the low energy structures found with the semi-empirical potential, it is also judicious to investigate structures with favourable geometrical properties, which are not necessarily found by the semi empirical search of the PES. In the present study, we also performed local relaxations, at the DFT level, of cubic ( $\mathrm{n}=9$ and 35), cuboctahedral $(\mathrm{n}=13)$, decahedral $(\mathrm{n}=13)$, rhombic dodecahedral $(n=15)$, octahedral $(n=19)$, one and four atom-truncated tetrahedral $(n=$ 9, 16, 19, 31 and 34), one atom capped-tetrahedral $(\mathrm{n}=11,21$ and 36), tetrahedral $(\mathrm{n}=$ 10, 20 and 35) structures as well as the recently reported 32 atom fullerene [14] structure. In addition to these ordered structures, we finally investigated planar structures up to $\mathrm{n}=$ 14, including the global minima reported by Hakkinen et al [13], Bonačić-Koutecký et al. [11] and Lee et al. [12]. 


\section{A. Empirical PES Searches}

We have implemented a Monte Carlo (MC) Simulated Anneal (SA) [15] method to search the empirical PES for low-energy structures. This is a well-established technique and the reader is refereed to the literature [16]. The starting cluster geometry is generated randomly with $N$ atoms distributed in a sphere of radius $\mathrm{r}_{\mathrm{s}}=2.5 \times N^{1 / 3} \AA$. Equilibration at each temperature is achieved when the total energy of the cluster, averaged over 100 consecutive moves, varies only within a defined energy tolerance for 10 consecutive cycles. The energy tolerance employed depends upon the potential used and cluster size. The temperature is then reduced by a factor $\mathrm{f}_{\mathrm{T}}=0.5$ and the process repeated. At each temperature cycle, the radius $r_{s}$ of the sphere in which the selected atom is moving is initially reduced by a factor $f_{r}=0.9$, and then readjusted [17] so that the acceptance/rejection ratio is kept between 0.4 and 0.6 . Ten temperature cycles are applied for a structure optimisation, and the initial temperature is selected so that the acceptance/rejection ratio initially lies between 0.4 and 0.6 . The annealing temperature was also controlled so that local minima could also be located as well as the global minimum. For each cluster size, $10^{4}$ random starting configurations were optimised with the SA method and the ten distinct lowest energy structures were locally relaxed at the DFT level.

The SA method is a robust method for minimising functions with many variables that, unlike steepest descent or a conjugate gradient methods, can escape from shallower minima to locate deeper minima, and under careful annealing conditions find the global minimum. Another attractive feature of the SA is that the global minimum is found 
through a "logical" sequence, where each intermediate low energy configuration is a refinement of a system previously equilibrated at a higher temperature. The use of a Monte Carlo method starting at completely random structures also has the obvious advantage that the final minimum structures are (relatively) unbiased by the choice of starting structure. However, due to the random nature of searching, a large number of potential energy evaluations are required to locate the global minium. As shown by Zeiri [18], Genetic Algorithms (GA), which are commonly used nowadays, [19] [20] [6], can require only 1/10 the number of energy evaluations required by SA methods. For clusters sizes in the present study this is not such a problem, although larger clusters will likely require a GA method to remain tractable.

The atomic interactions were modelled by a Lennard-Jones [21] pair potential for $\mathrm{Au}_{\mathrm{n}}, \mathrm{n}=3$ to 30, and an $n$-body Gupta [22] potential for $\mathrm{Au}_{\mathrm{n}}, \mathrm{n}=20$ to 38. The standard 6/12 form for the Lennard-Jones potential was used with a well depth, $\varepsilon=4.4592 \mathrm{eV}$, and a zero value of the potential, $\sigma=2.1644 \AA$, obtained by fitting an $a b$ initio calculation of the PES for the gold dimer (see section II.B).

Clearly, a two-body potential may not provide an adequate model of metallic clusters, particularly as the cluster size increases. However, it is arguable whether a metallic behaviour occurs for such small cluster sizes, and besides we are more concerned in using it to find low energy candidates for the $a b$ initio optimisations, rather than predicting the actual global minimum with the SA. Lennard-Jones potentials, and pair potentials in general, are easy to implement and computationally cheap.

For larger clusters we use the n-body Gupta potential parameterised by Cleri and Rosato [22], which successfully reproduces fcc bulk cohesive energy, lattice parameter 
and elastic constants. The reader is again refereed to the literature for details [22]. The underlying method, the tight-binding second moment approximation (TB-SMA), was originally designed for transition metals whose cohesive energy is related to large $d$-band densities of states and can be derived from the second moment. However, it was shown by Cleri et Rosato [22] that for noble metals, for which higher moments have to be included in the computation of the cohesive energy due to the $s$ - $d$ hybridisation, the TBSMA model can still be successfully applied by increasing the range of the potential. By attempting to treat metallic bonding in a reasonable way, these types of potentials appear to be appropriate to characterise large clusters exhibiting metallic behaviour. The main drawback of the n-body potentials is that they are computationally expensive because of their many-body character, and it is unclear how large the cluster must be in order for this description to be reasonable.

The LJ potential is seen to predict structures whose total energies are increasingly higher than the DFT low energy structures for $n \geq 20$, whereas the Gupta potential consistently predicts low energy structures lying close in energy with the DFT global minima (see fig. 9(a)). For this reason, the n-body Gupta potential was used for clusters with $n \geq 20$.

\section{B. Ab Initio Calculations}

Structure optimisations of the low energy candidates determined from empirical SA calculations, and the mapping of the PESs for clusters containing up to 5 atoms were performed using density functional theory (DFT) calculations. The $a b$ initio calculations were carried out using the SIESTA code $[7,23]$, which performs a fully self consistent 
DFT calculation based on the linear combination of atomic orbitals (LCAO) approximation. The DFT calculations were performed within the local spin density approximation (LSDA) as parameterised by Perdew and Zunger [24]. A pseudo-potential basis set was used with the core-electrons described by a relativistic Troullier-Martins [25] norm conserving pseudo-potential with cutoff radii $\left(r_{c}\right)$ of 2.24, 2.24, 2.32 and 1.48 bohr for the s, p, d and f orbitals, respectively. Relativistic effects are known to be important in the quantum chemistry of gold, resulting in contraction of the 6s orbital and strong s-d hybridisation [26]. A double-zeta basis set with d orbital polarisation was used to describe the valence $5 \mathrm{~d}$ and 6 s electrons. The energy cutoff of the equivalent plane wave was set to 100Ry. The tolerance criteria for self-consistency was a change in the value of density matrix smaller than $10^{-4}$. Structure optimisations were performed with an unconstrained Conjugate Gradient (CG) minimisation with a maximum atomic displacement of 0.2 bohr per step. The minimisation convergence was achieved with a force tolerance less than $0.04 \mathrm{eV} / \AA$.

The DFT calculations predict a cohesive energy $E_{c}=3.95 \mathrm{eV}$, a lattice parameter $a=4.07 \AA$ and a bulk modulus $B=205 \mathrm{GPa}$ for bulk fcc gold. These values agree well with the experimental values of $3.81 \mathrm{eV}, 4.08 \AA$ and $173 \mathrm{GPa}$ respectively reported in the literature [27]. The calculated ionisation potential and the electron affinity of atomic gold, $9.78 \mathrm{eV}$ and $2.45 \mathrm{eV}$, respectively, were also found to be in agreement with the experimental values, $9.22 \mathrm{eV}$ [28] and $2.31 \mathrm{eV}$ [29]. Calculations performed on the dimer yielded a bond length of $2.43 \AA$ and a binding energy of $2.78 \mathrm{eV}$. These values are also in reasonable agreement with the experimental values, $2.47 \AA$ [30] and $2.30 \mathrm{eV}$ [31], respectively. 


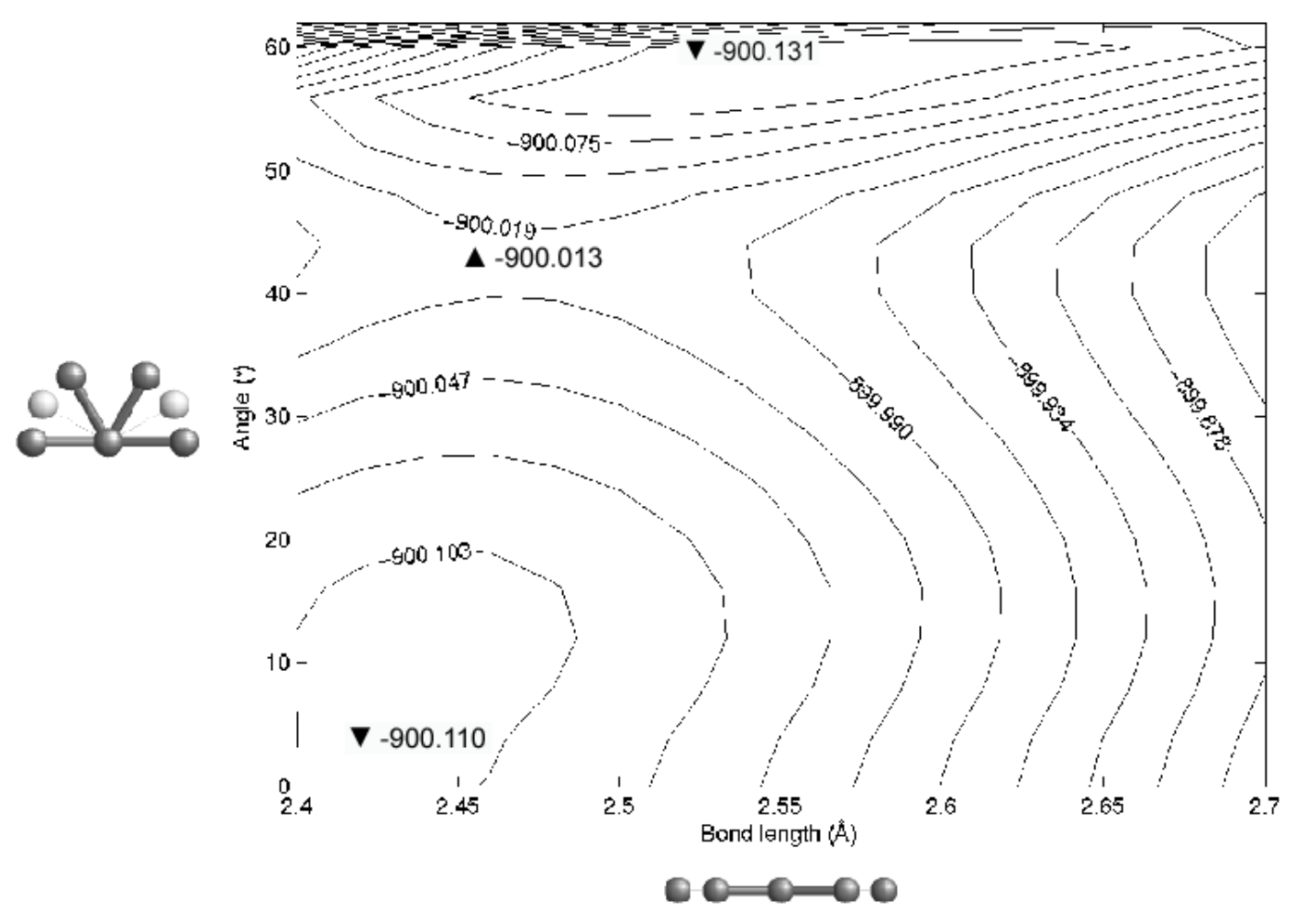

Fig. 1. Potential Energy Surface of $\mathrm{Au}_{3}$.

\section{RESULTS AND DISCUSSION}

\section{A. Potential Energy Surfaces for $A u_{n}(n=3$ to 5)}

The DFT PES of $\mathrm{Au}_{3}$ is shown in figure 1. The transition between the linear trimer and isosceles triangle was mapped by folding the molecule along its centre (see fig. 1). At a relatively course level the $\mathrm{Au}_{3}$ PES appears to contain two wells, the deeper one corresponding to an equilateral triangle, and the shallower corresponding to an obtuse triangle. The shallow minimum corresponds to an obtuse triangle with an obtuse angle of $170.78^{\circ}$ and short bond lengths of $2.43 \AA$. A refined scan of the PES around the equilateral triangle revealed two local minima: an acute triangle with an acute angle of $59.2^{\circ}$ and long bond lengths of $2.59 \AA$, and another obtuse triangle with obtuse angle of $65^{\circ}$ and short bond lengths of $2.53 \AA$. The obtuse triangle is $6 \times 10^{-4} \mathrm{eV} /$ atom lower in 


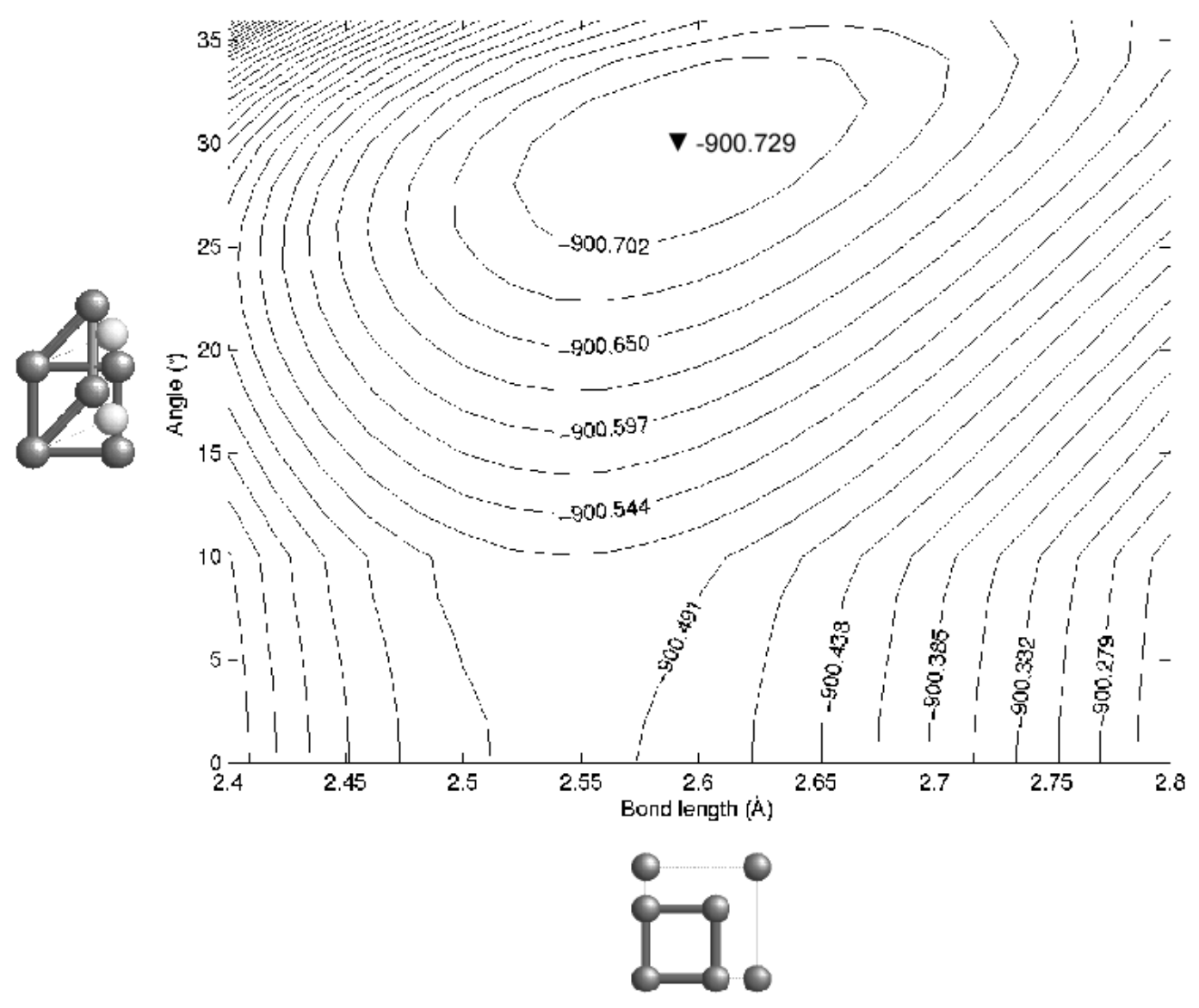

Fig. 2. Partial Potential Energy Surface between the square structure and the planar rhombus for $\mathrm{Au}_{4}$.

energy than the acute triangle, and the activation barrier from this minimum to the acute triangle is $10^{-3} \mathrm{eV} /$ atom. Both the linear form [8,32] and obtuse triangle [10,33] have been found previously as the global minimum. Wesendrup et al. [34] have shown that the lowest energy structure of the trimer is very sensitive to the method used. They find an acute triangle at relativistic- $\mathrm{HF}$ and $-\operatorname{CCSD}(\mathrm{T})$ levels and an obtuse triangle at a relativistic-MP2 level. The PES in figure 1 indicates that the potential barrier is very steep towards acute angles but shallower on the obtuse side. The bond lengths for both our structures are somewhat shorter than previous results, Wang et al [8] calculate a 


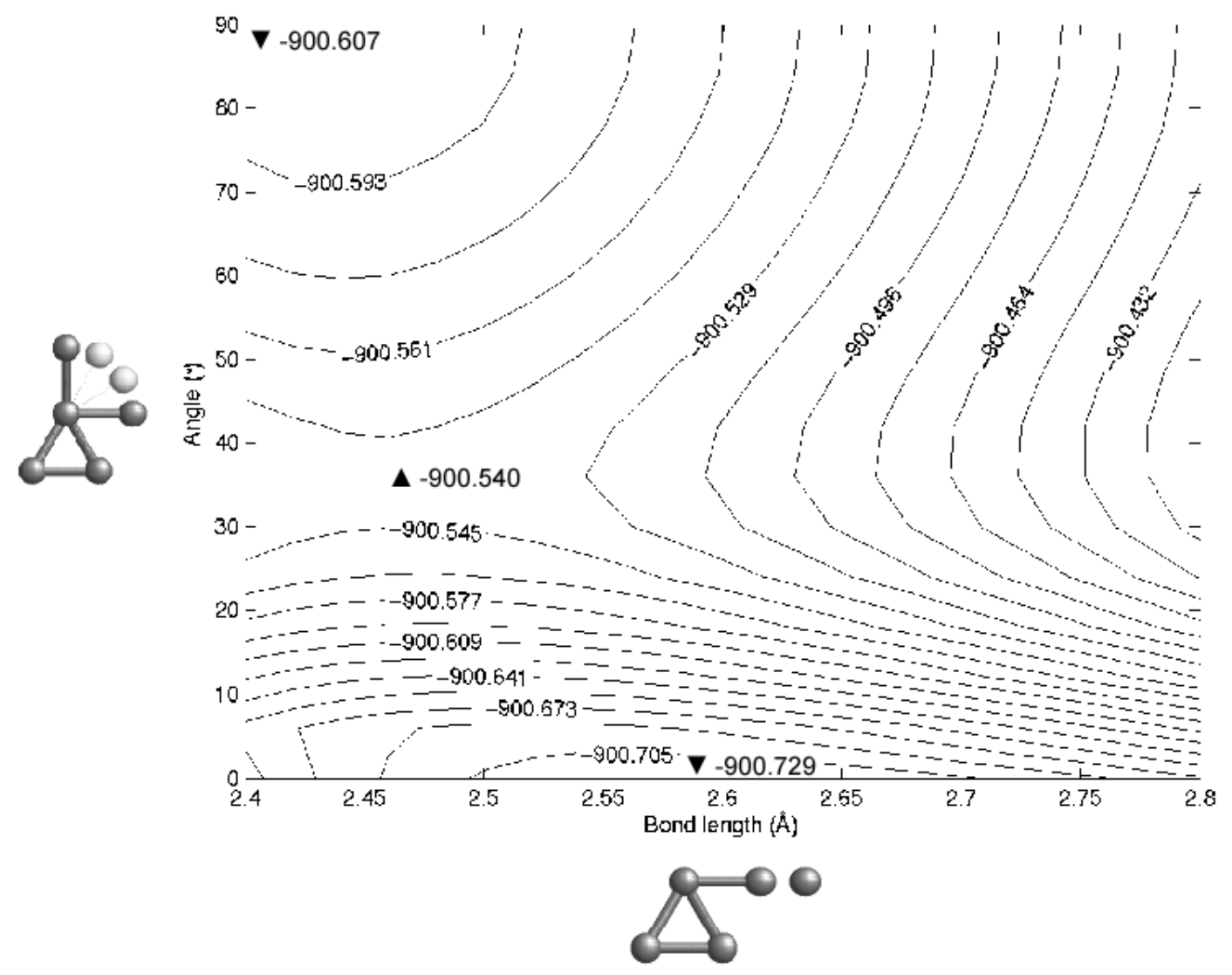

Fig. 3. Partial Potential Energy Surface between the "Y-shaped" structure and the planar rhombus for $\mathrm{Au}_{4}$.

value of $2.67 \AA$ for the linear trimer, using the LDA functional and Hakkinen et al [10] a short bond length of $2.63 \AA$ for the obtuse triangle with an obtuse angle of $66.7^{\circ}$ with the GGA functional. From our PES the activation barrier from the ground state structure to the large obtuse angled triangle is $0.118 \mathrm{eV} /$ atom (see fig. 1).

The PES for the 4 atom cluster is shown in figures 2, 3 and 4 . These three surfaces show transitions between the planar rhombus and square, Y-shaped and 3D tetrahedron respectively. The planar rhombus being the global minimum with bond lengths of $2.59 \AA$ and angles of $60^{\circ}$. Previous studies also find this as the lowest energy structure $[8,10]$, although Hakkinen et al give a bond length of $2.70 \AA$, again exceeding 


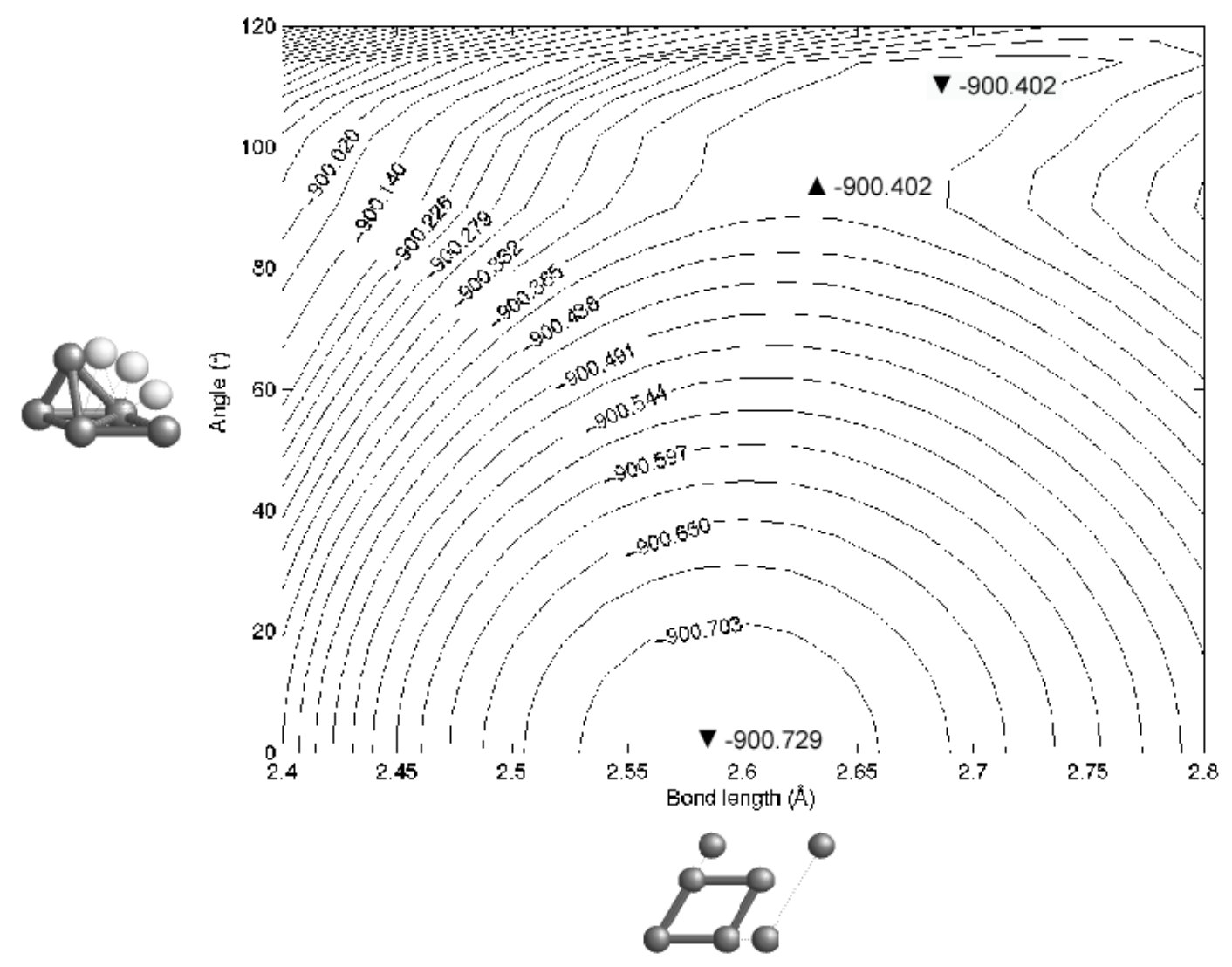

Fig. 4. Partial Potential Energy Surface between the 3D tetrahedron and the planar rhombus for $\mathrm{Au}_{4}$.

our value, and an angle of $58.9^{\circ}$, which is a little lower than our value. Out of these 4 structures only the planar rhombus and Y-shaped structure represent minima, the square and 3D tetrahedron being transition states. The planar rhombus is $0.122 \mathrm{eV} /$ atom more stable than the Y-shaped structure, about half the value obtained by Wang et al [8]. The activation energy from the planar rhombus is about $0.189 \mathrm{eV} /$ atom. The energy difference between the planar rhombus and 3D tetrahedron is only $0.327 \mathrm{eV} / \mathrm{atom}$, Wang et al [8], by contrast give a value that exceeds $1.5 \mathrm{eV} /$ atom. The sensitivity of the optimum structure to the method used for the 4 atom cluster has also been shown by 


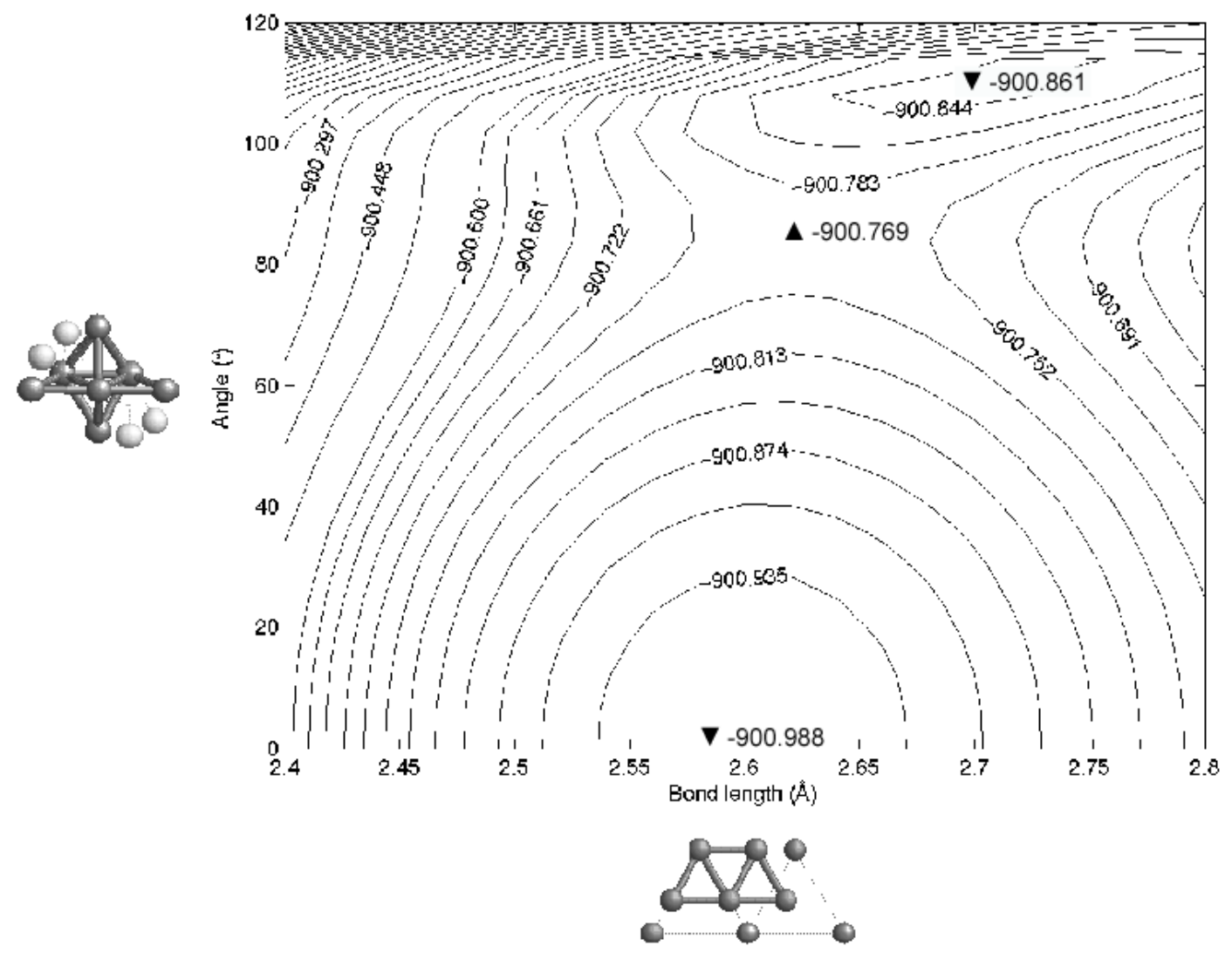

Fig. 5. Partial Potential Energy Surface between the "W-shaped" structure and the trigonal bipyramid for $\mathrm{Au}_{5}$.

Gronbech et al [33], where the planar rhombus is more stable using the LSDA functional while the "Y-shaped” structure is more stable with the BLYP functional.

Finally, a portion of the PES for the 5 atom cluster is shown in figure 5, corresponding to the transition between the trigonal bipyramid and the planar "Wshaped” structure. Both structures are minima separated by an energy difference of 0.127 eV/atom, the planar structure being the global minimum, the energy barrier is 0.219 eV/atom from the planar structure. By contrast to the previous clusters, the w-shaped planar structure seems to be the global minimum irrespective of the level of theory employed $[8,10,33,35]$. The energy difference between these two low energy structures is again considerably smaller than that reported by Wang et al [8], $0.79 \mathrm{eV} / \mathrm{atom}$, and 
bond lengths are smaller than those reported by Hakkinen et al [10]. Our relaxed structure is a trapezoid with a base of three Au atoms each separated by $2.54 \AA$, the three remaining outside bond lengths are $2.58 \AA$ and the inside bond lengths are $2.72 \AA$, as opposed to base and inside bond lengths of $2.64 \AA$ and $2.78 \AA$ from Hakkinen et al [10].

\section{B. Structure of Larger Clusters ( $n=6$ to 38$)$}

Optimum DFT structures for clusters in the range of 6 to 38 atoms are shown in figures 6, 7 and 8 . These were obtained by generating 10 low energy candidates by our empirical SA method and relaxing using a conjugate-gradient DFT calculation. In addition to the semi-empirical structures, we also performed DFT local relaxation on ordered structures (see section II). In most cases the energy ordering of the low energy isomers differs at the empirical and $a b$ initio levels.

The Lennard-Jones potential was used for the empirical portion of the calculations for clusters below 31 atoms. The lowest energy structures generated by this potential agree with those reported in the Cambridge Cluster Database [36], giving us confidence that our implementation of the SA is behaving correctly.

For the smaller clusters in this size range there is still some disagreement about where the transition occurs between 2D and 3D structures. While there is little dispute that the $\mathrm{Au}_{6}$ cluster is a planar triangular structure $[8,10,11,37]$, variance exists for the $\mathrm{Au}_{7}$ structure. Our calculations predict this as the first 3D structure, the pentagonal bipryamid (7, fig. 6) being the most stable configuration. Hakinnen et al. [10] predict a hexagonal planar structure (7b, fig. 6). Bonačić-Koutecký 


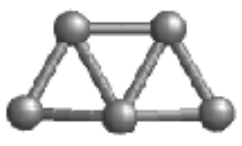

5

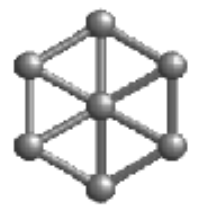

$7 \mathbf{b}-\Delta \mathrm{E}=0.069$

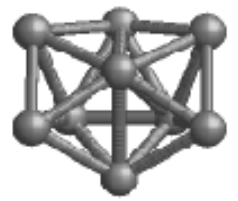

9a- $-\Delta \mathrm{E}=0$

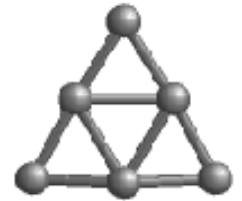

6

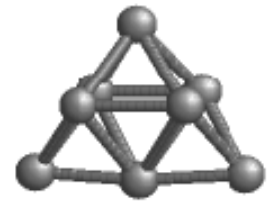

8

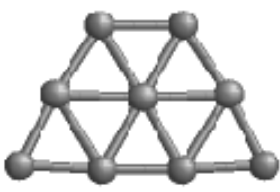

$9 b-\Delta E=0.069$

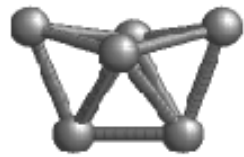

$\mathbf{6 a}-\Delta \mathrm{E}=0.178$

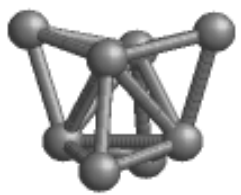

$\mathbf{8} \mathbf{a}-\Delta \mathrm{E}=0.021$

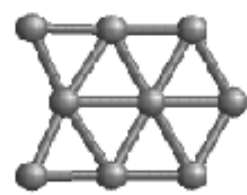

$9 c-\Delta E=0.069$
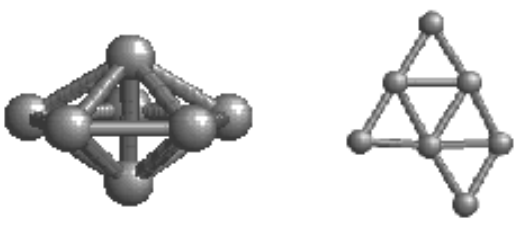

7

$7 \mathbf{a}-\Delta \mathrm{E}=0.041$

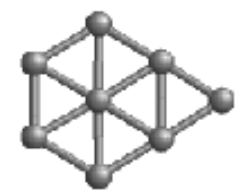

$\mathbf{8 b}-\Delta \mathrm{E}=0.067$

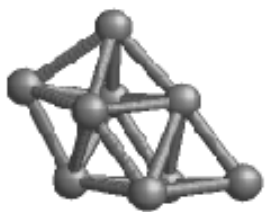

9
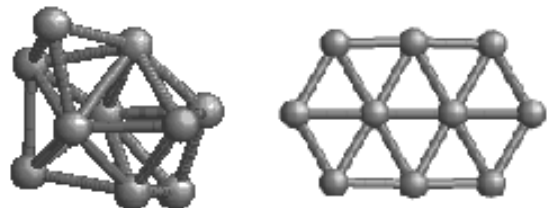

10

$10 \mathrm{a}-\Delta \mathrm{E}=0.046$

Fig. 6. Low energy structures of $\mathrm{Au}_{\mathrm{n}}, \mathrm{n}=5-10$. The clusters with letter indices correspond to isomers. $\Delta \mathrm{E}$ is the difference in total energy per atom $(\mathrm{eV})$ between the isomer and the ground state structure.

et al. [11] and Lee et al. [12] also report a planar geometry: a one atom capped triangle structure (7a, fig. 6). In contrast, Wang et al [8] find the same 3D structure as our own, albeit with an energy difference that is about twice the value found here of $\underline{0.069}$ eV/atom. It is important to note that the $3 \mathrm{D}$ structure is predicted by LDA calculations while the planar structures are the result of GGA calculations.

For clusters containing between 7 and 10 atoms although the lowest energy structures are all 3D (see fig. 7) there are a number of planar isomers within 0.069 eV/atom or less. Again this is in disagreement with Hakinnen et al. [13], BonačićKoutecký et al. [11] and Lee et al. [12] who report planar structures within this size range. However, it is important to note that the GGA calculations by Lee et al. [12] also 
predict a small difference in energy, up to $0.070 \mathrm{eV}$ per atom, in favour of the planar structures. A combination of ion mobility measurements and GGA-DFT calculations of cationic gold clusters by Gilb et al. [38], shows that GGA lowest energy structures are compatible with experimental cross sections, which tend to support the planar GGA structures over the 3D LDA structures for $\mathrm{Au}_{\mathrm{n}}$ with $\mathrm{n}>6$. On the other hand, a similar investigation on anionic gold clusters by Furche et al. [39] shows that GGA overestimates the stability of planar structures by predicting these to be the minimum up to $\mathrm{Au}_{15}{ }^{-}$whereas $\mathrm{Au}_{13}{ }^{-}$is found to be three dimensional. The variety of ground state structures reported for $\mathrm{Au}_{3}, \mathrm{Au}_{4}, \mathrm{Au}_{7}$ to $\mathrm{Au}_{10}$ suggests that not only relativistic effects [40] but also the level of theory used strongly influences the stability. Nonetheless it is debateable whether the lowest energy structure can be unambiguously identified within different methods given the small energy difference between planar and 3D structures predicted by LDA and GGA [12]. Finally, we want to stress that two isomers are found as the ground structure for $\mathrm{Au}_{9}$ (9 and 9a, fig. 6).

Beyond $\mathrm{Au}_{10}$ 3D structures dominate. As shown in figure 9(a), the energy difference between the planar lowest energy structure and the global minimum increases considerably beyond $n=10$. Similarly to Aug, two different structures are found for the $\mathrm{Au}_{11}$ global minimum (11 and 11a, fig. 7). Up to 16 atoms the structures are flat cage like structures with no internal atoms, more importantly they are all disordered. The symmetric icosahedral structure for $\mathrm{Au}_{13}$ (13a, fig. 7) predicted by a simple LennardJones potential as the lowest energy structure lies about $0.148 \mathrm{eV} / \mathrm{atom}$ above a disordered structure (13, fig. 7) at the DFT level. 


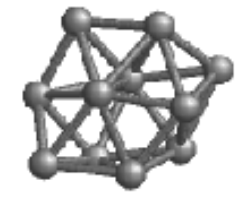

11

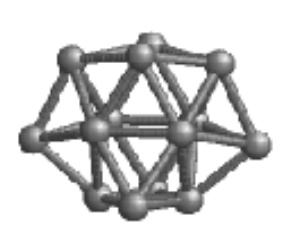

14

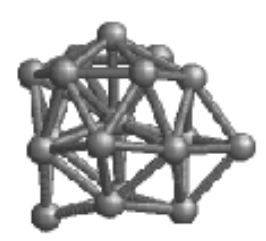

19

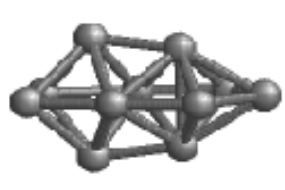

11a $-\Delta \mathrm{E}=0$

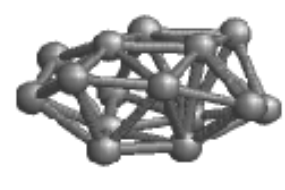

15

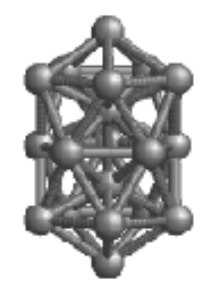

19a $-\Delta \mathrm{E}=0.090$

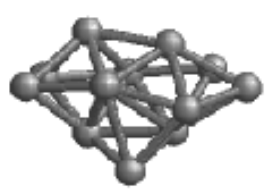

12

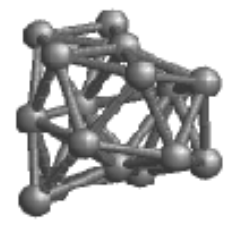

16

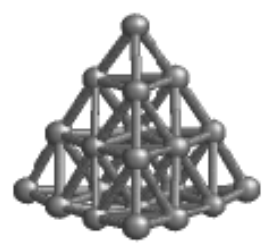

20

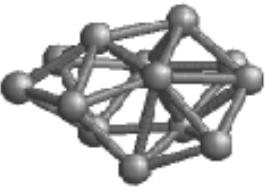

13

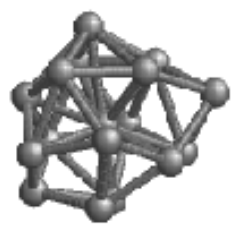

17

18

Fig. 7. Low energy structures of $\mathrm{Au}_{\mathrm{n}}, \mathrm{n}=11-20$. The clusters with letter indices correspond to isomers. $\Delta \mathrm{E}$ is the difference in total energy per atom $(\mathrm{eV})$ between the isomer and the ground state structure.

The transition between flat cage and compact structures is found to occur at $\mathrm{n}=$

16, at which the lowest energy structure is neither a flat cage structure nor a compact structure, and is only composed of surface atoms. The first structure with an internal atom appears at $\mathrm{Au}_{17}$, and has $\mathrm{C}_{2 \mathrm{v}}$ symmetry. The lowest energy structures of $\mathrm{Au}_{18}$ and $\mathrm{Au}_{19}$ are derived from this latter structure. $\mathrm{Au}_{18}$ undergo distortions due to the inclusion of the additional atoms, while $\mathrm{Au}_{19}$ exhibits the same structure as $\mathrm{Au}_{17}$ with two capping atoms. Once again there are a number of isomers lying close in energy, with about $75 \%$ of the relaxed structures lying within $0.05 \mathrm{eV} /$ atom of the ground energy.

For $n \geq 20$, the n-body Gupta potential [22] was used to map the PES with the SA method (LJ potential calculations have additionally been performed up to $n=30$ ). The 
Gupta lowest energy structures of $\mathrm{Au}_{20}$ to $\mathrm{Au}_{38}$ found with the SA were identical to the structures reported by Darby et al [41], except for $\mathrm{Au}_{33}$. The SA revealed a structure with a $\mathrm{C}_{2 \mathrm{v}}$ symmetry (33G, fig. 8) that is $7.488 \times 10^{-3} \mathrm{eV}$ lower in energy (at the Gupta potential level) than the structure reported previously [41].

The ground structure of $\mathrm{Au}_{20}$ is found to be tetrahedral (20, fig 7) in agreement with previous experimental and theoretical studies [9,42]. The tetrahedron was not predicted as one of the ten lowest energy structures by the SA with either the Gupta or the LJ potential. With the Gupta implementation of the potential, the tetrahedron is found to be $1.198 \mathrm{eV}$ higher in energy than the Gupta lowest energy structure (see Darby [41]). Similarly, with the LJ implementation, the tetrahedron is found to be a local minimum that is higher in energy than the $\mathrm{LJ} \mathrm{Au}_{20}$ global minimum. The $\mathrm{Au}_{20}$ tetrahedron is 0.033 eV/atom lower in energy than the more stable disordered structure (20a, fig. 7) at the DFT level. The other possible tetrahedral structures within our size range, $\mathrm{Au}_{10}$ and $\mathrm{Au}_{35}$, are respectively 0.088 and $0.121 \mathrm{eV} /$ atom higher in energy than the disordered lowest energy structures. The other tetrahedral based structures: the four atom truncated tetrahedrons, $\mathrm{Au}_{16}$ and $\mathrm{Au}_{31}$, the one atom truncated tetrahedrons, $\mathrm{Au}_{9}, \mathrm{Au}_{19}$ and $\mathrm{Au}_{34}$, and the one atom capped tetrahedrons (including different isomers with a capping atom in distinct location on the (111) surface), $\mathrm{Au}_{11}, \mathrm{Au}_{21}$ and $\mathrm{Au}_{36}$, are also found to be less energetically favourable than the corresponding disordered structures. All these tetrahedral based structures are at least $0.013 \mathrm{eV} /$ atom higher in energy than our global minima except for $\mathrm{Au}_{16}$ and $\mathrm{Au}_{9}$ that both lie within $0.003 \mathrm{eV} /$ atom of the corresponding lowest energy structures (9, 9a, fig. 6, and 16, fig. 7). 


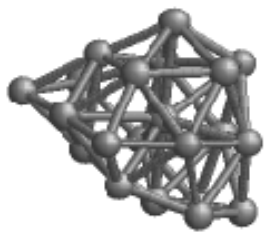

21

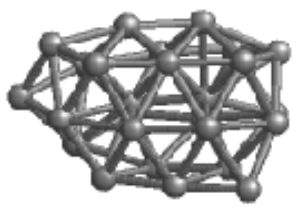

26

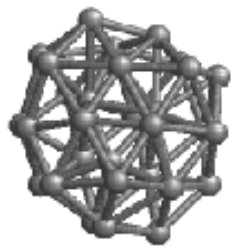

30

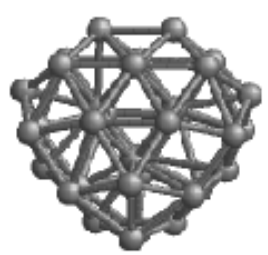

34

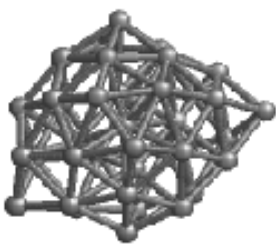

38

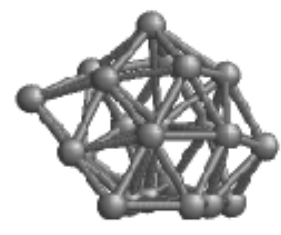

22

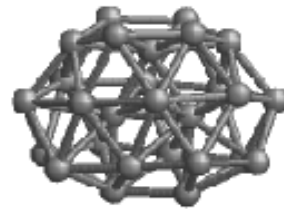

27

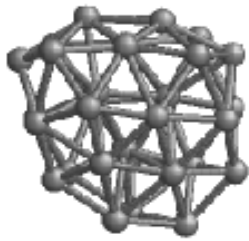

31

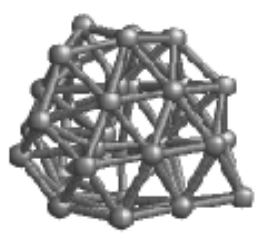

35

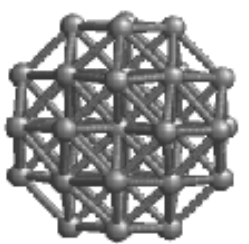

$38-\Delta \mathrm{E}=0.026$

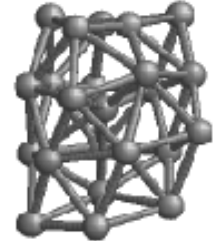

23

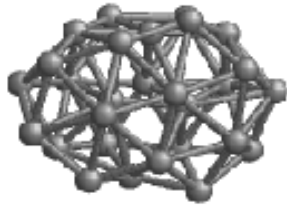

28

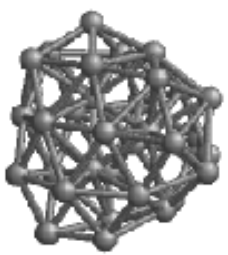

32

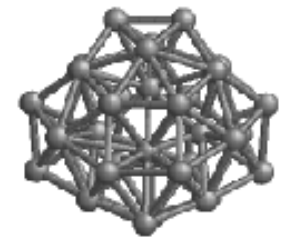

36

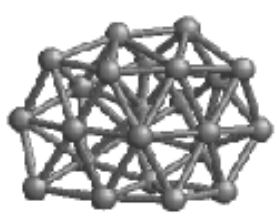

24

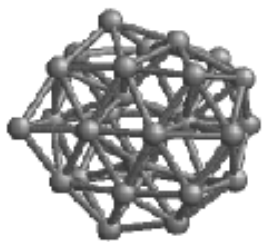

29

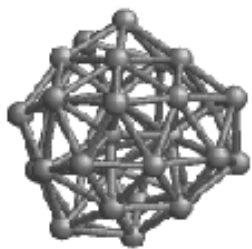

33

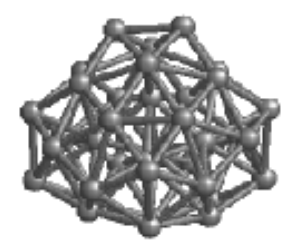

37

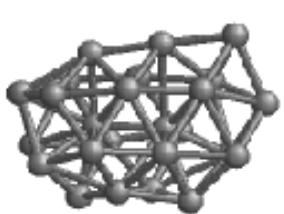

25

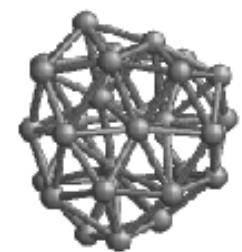

$29 \mathrm{a}-\Delta \mathrm{E}=0$

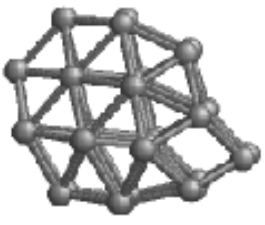

33G

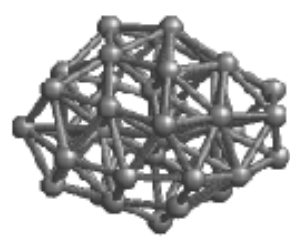

$\mathbf{3 7} \mathbf{a}-\Delta \mathrm{E}=0$

Fig. 8. Low energy structures of $\mathrm{Au}_{\mathrm{n}}, \mathrm{n}=21-38$. The clusters with letter indices correspond to isomers. $\Delta \mathrm{E}$ is the difference in total energy per atom $(\mathrm{eV})$ between the isomer and the ground state structure. The 33G cluster represents the Gupta Au33 global minimum (see text).

Rather than describe each of the DFT relaxed low energy structures for clusters containing more than 20 atoms we again refer the reader to the structures shown in figure 
8, and note that they are all, to a lesser or greater extent, disordered. In addition, for $\mathrm{n}=$ 29 and 37 we find two structures with the same energy, and for all other structures find that almost all of the relaxed structures lie within $0.05 \mathrm{eV} /$ atom of each other. In agreement with Soler et al. [7], a disordered structure (38, fig. 8) is predicted as the $\mathrm{Au}_{38}$ lowest energy structure, $0.026 \mathrm{eV} /$ atom lower in energy than the truncated octahedron (38a, fig. 8) found with the Gupta potential.

We finally want to stress that the $\mathrm{Au}_{20}$ tetrahedron is the only ordered structure that was identified as a global minimum. The relaxation of the $\mathrm{Au}_{32}$ fullerene structure reported by Johansson et al. [14] also yielded a rhombic triacontahedron of approximately $8.7 \AA$ in diameter, but with a total energy $0.095 \mathrm{eV} /$ atom higher than the disordered $\mathrm{Au}_{32}$ ground structure. The additional ordered structures that were relaxed at the DFT level but not mentioned in this section were found to be at least $0.05 \mathrm{eV} / \mathrm{atom}$ higher in energy than the corresponding disordered structures.

\section{Trends in Binding Energy and Electronic Structure}

The calculated bulk relative total energy per atom, $E_{R}(n)$, second differences in the total energy per atom, $\Delta^{2} E(n)$, and the highest occupied and lowest unoccupied molecular orbital (HUMO-LUMO) energy gap, $E_{H-L}(n)$, as a function of the cluster size are reported in Fig. 9 (a), (b) and (c), respectively. The relative total energy per atom of each cluster is computed as the difference between the total energy per atom of the bulk and the total energy per atom of the cluster: $E_{R}(n)=E_{\text {bulk }}-E_{\text {total }}(n)$. We also report in Fig. 9 (a) the relative total energies per atom of the planar, LJ and Gupta ground state structures at the DFT level. The LJ and Gupta minima that are missing at some sizes ( $\mathrm{n}=15,16,17,18$, 


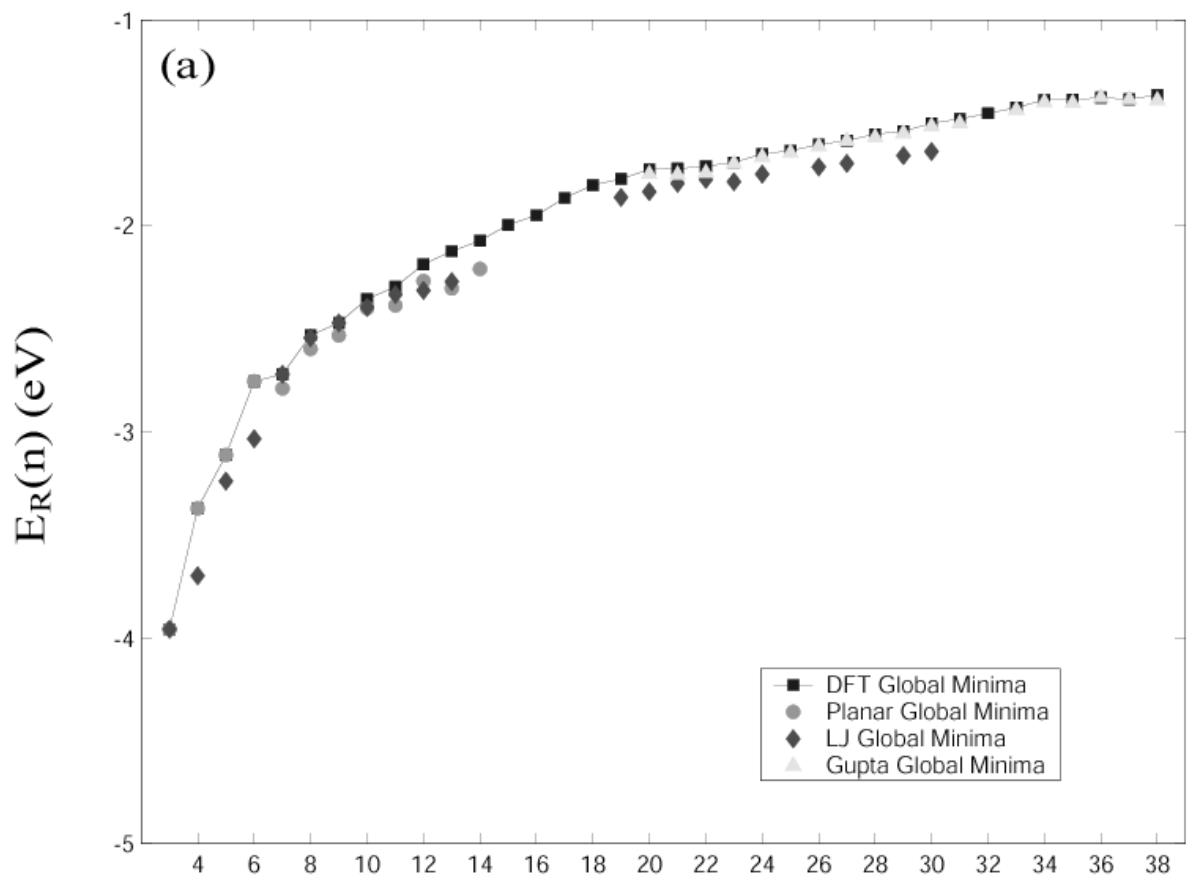

Cluster size (atoms)

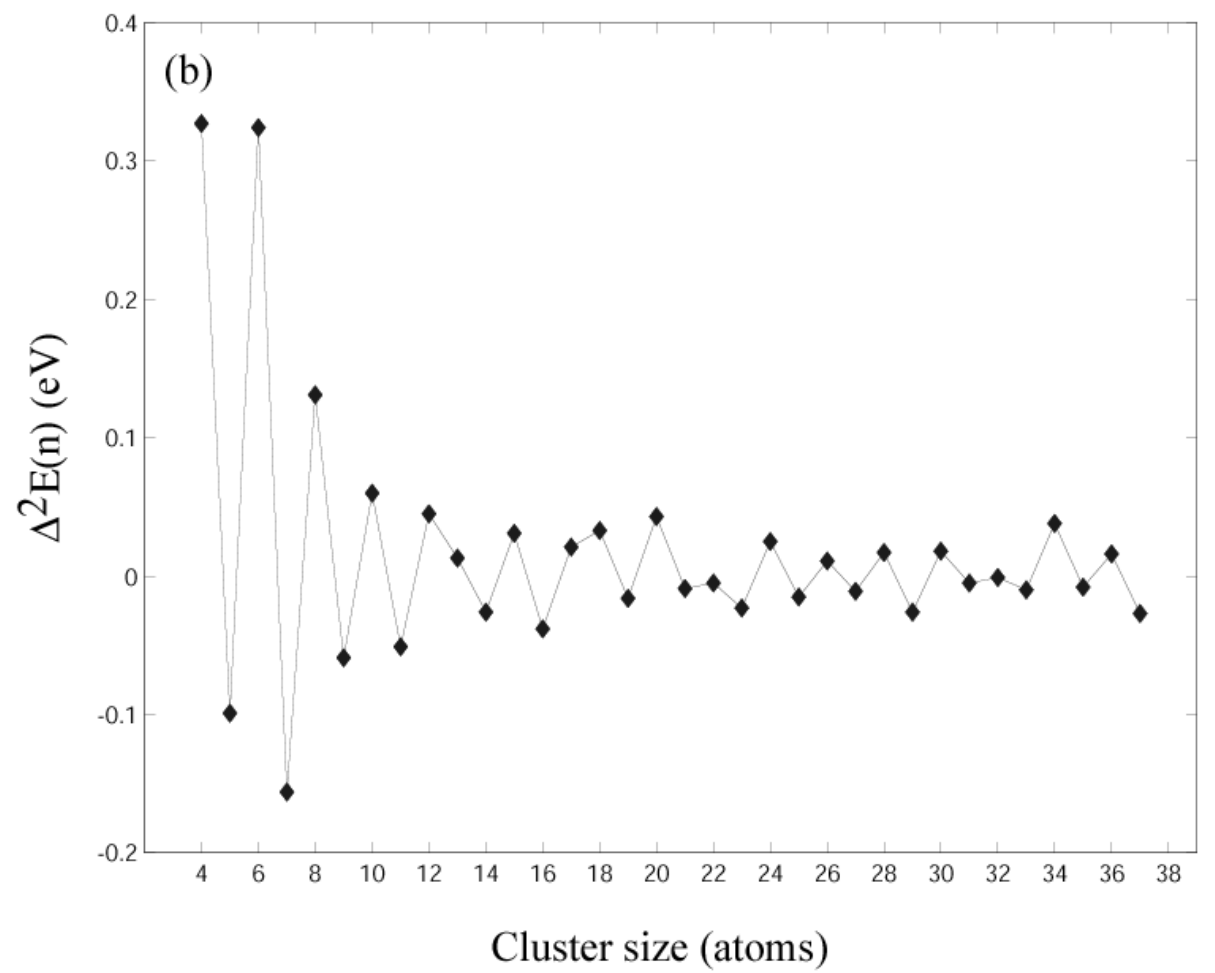




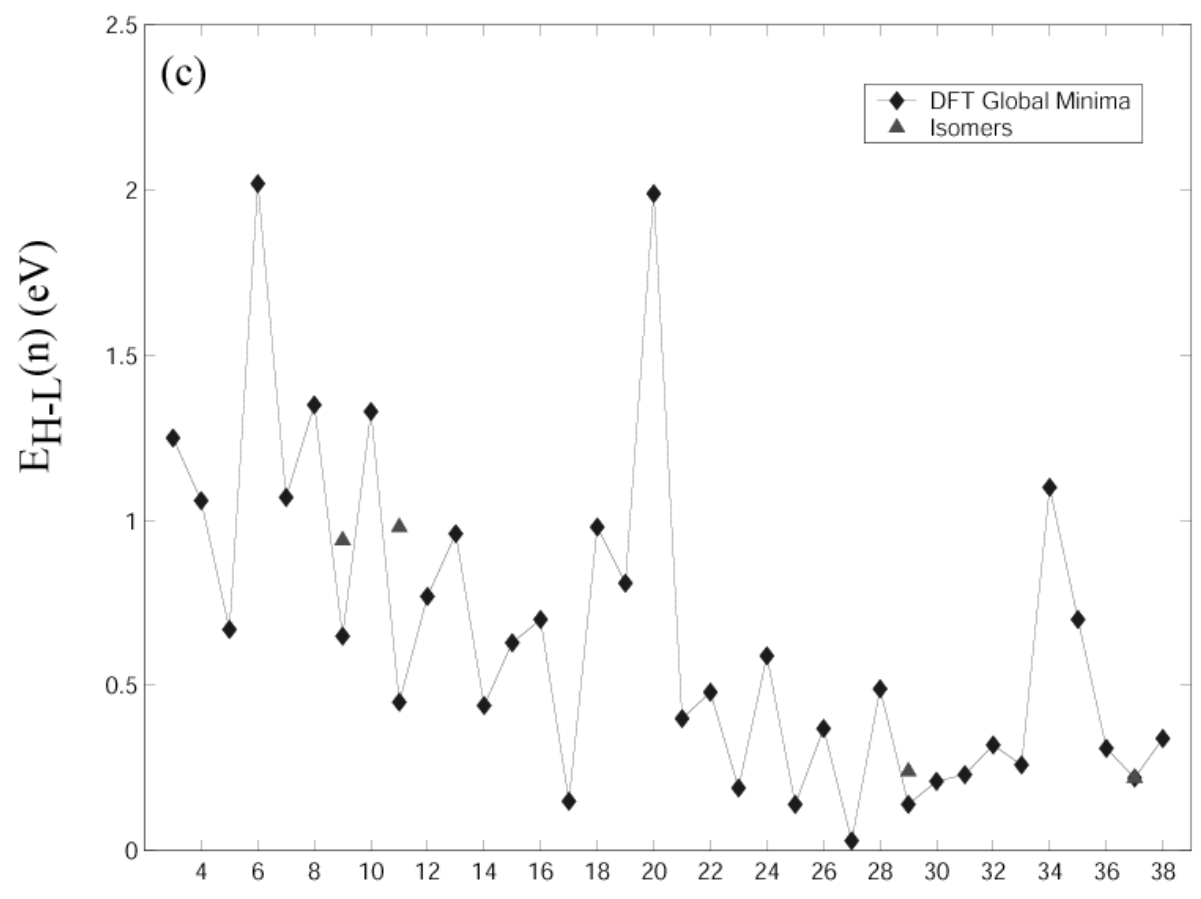

Cluster size (atoms)

Fig. 9. (a) Bulk relative total energy per atom, $E_{R}(n)$, (b) second differences in energy per atom, $\Delta^{2} E(n)$, and (c) HOMO-LUMO energy gap, $E_{H-L}(n)$, as a function of the cluster size.

25 and 28 for LJ, and $n=32$ for Gupta) correspond to transition structures at the DFT level and were relaxed to a different structure. The second differences in the total energy per atom were computed as $\Delta^{2} E(n)=E_{\text {total }}(n+1)+E_{\text {total }}(n-1)-2 E_{\text {total }}(n)$.

The relative total energy per atom is seen to increase gradually as a function of the cluster size (see Fig. 9(a)), and slowly tends towards 0, i.e. the total energy of the bulk. A change in the relative slope is observed between $\mathrm{Au}_{6}$ and $\mathrm{Au}_{7}$ at the transition from planar to $3 \mathrm{D}$ structures. Although the aim of this work is not to investigate the accuracy of the semi empirical potentials used, it is nonetheless interesting to note that the $\mathrm{LJ}$ potential predict the ground structures for $\mathrm{Au}_{7}$ and $\mathrm{Au}_{9}$, and the Gupta potential for 
$\mathrm{Au}_{36}$. Fig. 9(a) also shows that the Gupta potential performs better than the LJ potential for $n \geq 20$.

Both $\Delta^{2} E(n)$ and $E_{H-L}(n)$ curves exhibit almost periodical odd-even oscillations as shown in Fig. 9 (b) and (c). Clusters containing an even number of atoms are seen to be more stable than their odd-numbered neighbours, except within the size range $\mathrm{n}=13$ to 17 (see Fig. 9 (b)). A similar trend is observed for the HUMO-LUMO gaps. The even numbered clusters have larger values than the neighbouring odd ones, except for $n=3$, 13, 14, 15, 31, 35 and for the $\mathrm{Au}_{11}$ and $\mathrm{Au}_{29}$ isomers (see Fig. 9(c)). This odd-even alternation is consistent with electron pairing. Even numbered clusters have their orbitals fully occupied giving them more stability than the odd numbered clusters that have the HOMO singly occupied. The values of the HOMO-LUMO energy gap as well as the amplitude of the oscillations are seen to decrease as the cluster size increases except within the vicinity of $\mathrm{Au}_{20}$ and $\mathrm{Au}_{34}$ where local maxima are observed. This decrease in the energy gap and in the amplitude of the oscillations suggests a transition towards a metallic behaviour. The large values of $E_{H-L}$ observed for $\mathrm{Au}_{6}, E_{H-L}(6)=2.02 \mathrm{eV}$, and $\mathrm{Au}_{20}, E_{H-L}(20)=1.99 \mathrm{eV}$, are in agreement with previous theoretical investigations by Hakkinen et al. [10] and Wang et al. [8] who reported 2.05 and $2.06 \mathrm{eV}$ for $\mathrm{Au}_{6}$ and by $\mathrm{Li}$ et al. [9] who reported $1.82 \mathrm{eV}$ for $\mathrm{Au}_{20}$, in agreement with their approximate experimental measure of $1.77 \mathrm{eV}$. Experimental estimates of the vertical Electron Affinities (EA) [43] reveal large local oscillations of the $\mathrm{EA}$ at the vicinities of $\mathrm{Au}_{6}, \mathrm{Au}_{20}$ and $\mathrm{Au}_{34}$ suggesting large local values of HUMO-LUMO gaps at the sizes $\mathrm{n}=6,20$ an 34, which is also in agreement with the present investigation. An experimental investigation of CO oxidation on Au clusters [44] reported a band gap energy of 
approximately $0.7 \mathrm{eV}$ for 3D Au clusters of about $1.5 \mathrm{~nm}$ in diameter supported on a $\mathrm{TiO}_{2}$ surface. Although this band gap corresponds to larger clusters whose geometries are supposedly very different from the geometries investigated here, it is still interesting to compare with our largest cluster, $\mathrm{Au}_{38}$, whose approximate diameter is about $1 \mathrm{~nm}$, which has a $0.34 \mathrm{eV}$ HOMO-LUMO energy gap. The difference in the energy gap is probably due to the very specific structure of the 3D clusters observed by Valden et al. [44] that is only two atom layers in height. Indeed, at slightly larger sizes, Valden et al. found that two atomic layer clusters still have an energy gap of about $0.5 \mathrm{eV}$ whereas the ones with three atomics layers or greater have an energy gap below $0.3 \mathrm{eV}$. In addition to geometrical considerations, the well known trend of DFT-LDA to underestimate the HOMO-LUMO energy gap will also contribute to the significant difference between our $\mathrm{Au}_{38}$ value and the value reported by Valden et al. [44].

\section{IV.CONCLUSIONS}

In the present work, structures of small gold clusters, $n=3$ to 38 , were investigated using first principles calculations. Potential Energy Surfaces of $\mathrm{Au}_{3}, \mathrm{Au}_{4}$ and $\mathrm{Au}_{5}$ were directly mapped using DFT-LDA single point calculations. For $n>5$, semi empirical potentials were first used to search the PESs, and local DFT-LDA relaxations were then preformed to find the global minima. In addition to the low energy candidates revealed by the semi empirical potential, relevant ordered structures were also relaxed at the DFT-LDA level.

Planar structures are found as ground structures up to $n=6$, and are found to persist as relatively low-lying isomers up to $\mathrm{n}=10$. Similarly, previous GGA investigations predicted small differences in energy, but favoured planar over three 
dimensional structures. This suggests that the level of theory employed, as well as relativistic effects, influeneces the relative stability of planar versus 3D structures. The planar to 3D transition occurring between $\mathrm{Au}_{6}$ and $\mathrm{Au}_{7}$ is also revealed by a change in the slope of the bulk relative total energy curve. Ordered structures are found to be favourable energetically for $\mathrm{n} \leq 9$, however beyond this disordered structures dominate with the notable exception of the tetrahedral $\mathrm{Au}_{20}$. Among the considerable number of additional ordered structures that were investigated, the $\mathrm{Au}_{20}$ tetrahedron is only one identified as a ground state structure. The semi-empirical potentials failed to locate the ordered $\mathrm{Au}_{20}$ tetrahedron and the other DFT lowest energy structures with the exception of $\mathrm{Au}_{7}, \mathrm{Au}_{9}$ and $\mathrm{Au}_{36}$. Within the size range $\mathrm{n}=3$ to 38 , numerous isomers are found lying close in energy to the global minima, and for $n=9,11,29$ and 37, two distinct structures are identified as lowest energy structures. This suggests that a range of structures can co-exist at room temperature for a given cluster size. The even-odd

oscillations that were observed in the $\Delta^{2} E(n)$ and $E_{H-L}(n)$ curves are consistent with electron pairing effect. The values and the oscillations of the HOMO-LUMO gaps that are seen to decrease suggest a transition towards a metallic behaviour as the cluster size increases.

\section{ACKNOWLEDGEMENTS}

This work was supported by University of Technology. Computing facilities were accessed through the merit allocation schemes of the APAC National Facility and AC3 in NSW. B. Soulé de Bas acknowledges receipt of an International Postgraduate Research 
Scholarship and stipend from the Faculty of Science, UTS. The authors are indebted to

Richard Leow, AC3, for technical support.

\section{REFERENCES}

[1] G. J. Hutchings J. Catal. 96 (1985) 292; M. Haruta, T. Kobayashi, H. Sano, and M. Yamada Chem. Lett. (1987) 405.

[2] A. P. Alivisatos, K. P. Johnsson, X. Peng, T. E. Wilson, C. J. Loweth, M. P. Bruchez, and P. G. Shultz Nature (London) 382 (1996) 609; R. P. Andres, T. Bein, M. Dorogi, S. Feng, J. I. Henderson, C. P. Kubiak, W. Mahoney, R. G. Osifichin, and R. Reifenberger Science 272 (1996) 1323; C. A. Mirkin, R. L. Letsinger, R. C. Mucic, and J. J. Storhoff Nature (London) 382 (1996) 607; R. L. Whetton, J. T. Khoury, M. M. Alvarez, S. Murthy, I. Vezmar, Z. L. Wang, P. W. Stephens, C. L. Cleveland, W. D. Luedtke, and U. Landman Advanced Materials 5 (1996) 8.

[3] S. J. Oldenberg, J. B. Jackson, S. L. Westcott, and N. J. Halas Appl. Phys. Lett. 75 (1999) 2897.

[4] O. D. Haberlan, S.-C. Chung, M. Stener, and N. Rosch J. Chem. Phys. 106 (1997) 5189.

[5] I. L. Garzon, K. Michaelian, M. R. Beltran, A. Posada-Amarillas, P. Ordejon, and E. Artacho Phys. Rev. Lett. 81 (1998) 1600.

[6] k. Michaelian, N. Rendon, and I. L. garzon Phys. Rev. B: Condens. Matter 60 (1999) 2000.

[7] J. M. Soler, M. R. Beltran, K. Michaelian, I. L. Garzon, P. Ordejon, D. Sanchez-Portal, and E. Artacho Phys. Rev. B: Condens. Matter 61 (2000) 5771.

[8] J. Wang, G. Wang, and J. Zhao Phys. Rev. B: Condens. Matter 66 (2002) 035418.

[9] J. Li, X. Li, H.-J. Zhai, and L.-S. Wang Science 299 (2003) 864.

[10] H. Hakkinen and U. Landman Phys. Rev. B: Condens. Matter 62 (2000) R2287.

[11] V. Bona i-Kouteck, J. Burda, R. Mitri, M. Ge, G. Zampella, and P. Fantucci J. Chem. Phys. 117 (2002) 3120.

[12] H. M. Lee, M. Ge, B. R. Sahu, P. Tarakeshwar, and K. S. Kim J. Phys. Chem. 107 (2003) 9994.

[13] H. Hakkinen, B. Yoon, U. landman, X. Li, H.-j. Zhai, and L.-S. Wang J. Phys. Chem. 107 (2003) 6168.

[14] M. Johansson, D. Sundholm, and J. Vaara Angew. Chem., Int. Ed. Engl. 43 (2004) 2678.

[15] Kirkpatrick S, C. D. Gelatt, and M. P. Vecchi Science (1983) 671.

[16] N. Metropolis, A. W. Rosenbluth, M. N. Rosenbluth, and A. H. Teller J. Chem. Phys. 21 (1953) 1087.

[17] A. Corana, M. Marchesi, C. Martini, and R. S ACM Trans. on Math. Software 13 (1987) 262.

[18] Y. Zeiri Physical Review E 51 (1995) 2769.

[19] B. Hartke J. Phys. Chem. 97 (1993) 9973.

[20] D. M. Deaven and K. M. Ho Phys. Rev. Lett. 75 (1995) 288.

[21] J. E. Lennard-Jones Proc. R. Soc. London, A 106 (1924) 463.

[22] F. Cleri and V. Rosato Phys. Rev. B: Condens. Matter 48 (1993) 22.

[23] P. Ordejon, E. Artacho, and J. M. Soler Phys. Rev. B: Condens. Matter 53 (1996) R10441.

[24] J. Perdew and A. Zunger Phys. Rev. B: Condens. Matter 23 (1981) 5075.

[25] N. Troullier and J. L. Martins Phys. Rev. B: Condens. Matter 43 (1991) 1993.

[26] P. Pyykko Chemical Reviews 88 (1988) 563.

[27] C. Kittle, Introduction to Solid State Physics, 7th Edition ed. Wiley, New York, (1996).

[28] C. E. Moore, Ionization Potentials and Ionization Units Derived from the Analysis of Optical Spectra. National Bureau of Standards, Washington, DC, (1970).

[29] H. Hotop and W. C. Lineberger J. Phys. Chem. Ref. Data 14 (1985) 731.

[30] E. U. Condon and H. Odishaw, Handbook of Physics, 2nd Edition ed. McGraw-Hill, New York, (1972).

[31] J. Kordis, K. A. Gingerich, and R. J. Seyse J. Chem. Phys. 61 (1974) 5114.

[32] K. G. Balasubramanian and M. Z. Liao J. Chem. Phys. 86 (1987) 5587.

[33] H. Gronbech and W. Andreoni Chem. Phys. 262 (2000) 1. 
R. Wesendrup, T. Hunt, and P. Schwerdtfeger J. Chem. Phys. 112 (2000) 9356.

[36]

D. Kruger, H. Fuchs, R. Rousseau, D. Marx, and M. Parrinello J. Chem. Phys. 115 (2001) 4776. http://brian.ch.cam.uk/CCD.html.

[37] G. Bravo-Perez, I. L. Garzon, and O. Novaro J. Mol. Struct. 493 (1999) 225.

[38] S. Gilb, P. Weis, F. Furche, R. Ahlrichs, and M. Kappes J. Chem. Phys. 116 (2002) 4094.

[39] F. Furche, R. Ahlrichs, P. Weis, C. Jacob, S. Gilb, T. Bierweiler, and M. Kappes J. Chem. Phys. 117 (2002) 6982.

[40] H. Hakkinen, M. Moseler, and U. Landman Phys. Rev. Lett. 89 (2002) 033401.

[41] S. Darby, T. V. Mortimer-Jones, R. L. Johnston, and C. Roberts J. Chem. Phys. 116 (2002) 1536.

[42] J. Wang, G. Wang, and J. Zhai Chem. Phys. Lett. 380 (2003) 716.

[43] K. J. Taylor, C. L. Pettiette-Hall, O. Cheshnovski, and R. E. Smalley J. Chem. Phys. 96 (1992) 3319.

[44] M. Valden, X. Lai, and D. W. Goodman Science 281 (1998) 1647.

\section{FIGURE CAPTIONS}

Fig. 1. Potential Energy Surface of $\mathrm{Au}_{3}$.

Fig. 2. Partial Potential Energy Surface between the square structure and the planar rhombus for $\mathrm{Au}_{4}$.

Fig. 3. Partial Potential Energy Surface between the "Y-shaped" structure and the planar rhombus for $\mathrm{Au}_{4}$.

Fig. 4. Partial Potential Energy Surface between the 3D tetrahedron and the planar rhombus for $\mathrm{Au}_{4}$.

Fig. 5. Partial Potential Energy Surface between the "W-shaped" structure and the trigonal bipyramid for $\mathrm{Au}_{5}$.

Fig. 6. Low energy structures of $A u_{n}, n=5-10$. The clusters with letter indices correspond to isomers. $\Delta \mathrm{E}$ is the difference in total energy per atom $(\mathrm{eV})$ between the isomer and the ground state structure.

Fig. 7. Low energy structures of $\mathrm{Au}_{\mathrm{n}}, \mathrm{n}=11-20$. The clusters with letter indices correspond to isomers. $\Delta \mathrm{E}$ is the difference in total energy per atom $(\mathrm{eV})$ between the isomer and the ground state structure.

Fig. 8. Low energy structures of $\mathrm{Au}_{\mathrm{n}}, \mathrm{n}=21-38$. The clusters with letter indices correspond to isomers. $\Delta \mathrm{E}$ is the difference in total energy per atom $(\mathrm{eV})$ between the isomer and the ground state structure. The 33G cluster represents the Gupta Au33 global minimum (see text).

Fig. 9. (a) Bulk relative total energy per atom, $E_{R}(n)$, (b) second differences in energy per atom, $\Delta^{2} E(n)$, and (c) HOMO-LUMO energy gap, $E_{H-L}(n)$, as a function of the cluster size. 